\title{
Six Temperature Proxies of Scots Pine from the Interior of Northern Fennoscandia Combined in Three Frequency Ranges
}

\author{
Markus Lindholm, ${ }^{1}$ Maxim G. Ogurtsov, ${ }^{2}$ Risto Jalkanen, ${ }^{1}$ \\ Björn E. Gunnarson, ${ }^{3}$ and Tarmo Aalto ${ }^{1}$ \\ ${ }^{1}$ Metla, Rovaniemi Research Unit, P.O. Box 16, 96301 Rovaniemi, Finland \\ ${ }^{2}$ A.F. Ioffe Physico-Technical Institute, St. Petersburg 194 021, Russia \\ ${ }^{3}$ Bolin Centre for Climate Research, Department of Physical Geography and Quaternary Geology, Stockholm University, 10691 \\ Stockholm, Sweden
}

Correspondence should be addressed to Markus Lindholm; markus.lindholm@metla.fi

Received 20 December 2013; Revised 7 April 2014; Accepted 11 April 2014; Published 6 May 2014

Academic Editor: Silvio Gualdi

\begin{abstract}
Copyright (C) 2014 Markus Lindholm et al. This is an open access article distributed under the Creative Commons Attribution License, which permits unrestricted use, distribution, and reproduction in any medium, provided the original work is properly cited.
\end{abstract}

Six chronologies based on the growth of Scots pine from the inland of northern Fennoscandia were built to separately enhance low, medium, and higher frequencies in growth variability in 1000-2002. Several periodicities of growth were found in common in these data. Five of the low-frequency series have a significant oscillatory mode at 200-250 years of cycle length. Most series also have strong multidecadal scale variability and significant peaks at 33, 67, or 83-125 years. Reconstruction models for mean July and June-August as well as three longer period temperatures were built and compared using stringent verification statistics. We describe main differences in model performance $\left(R^{2}=0.53-0.62\right)$ between individual proxies as well as their various averages depending on provenance and proxy type, length of target period, and frequency range. A separate medium-frequency chronology (a proxy for June-August temperatures) is presented, which is closely similar in amplitude and duration to the last two cycles of the Atlantic multidecadal oscillation (AMO). The good synchrony between these two series is only hampered by a 10-year difference in timing. Recognizing a strong medium-frequency component in Fennoscandian climate proxies helps to explain part of the uncertainties in their 20th century trends.

\section{Introduction}

Several recent studies have discussed the potential of highresolution proxies based on the growth of Scots pine from northern Fennoscandia for reconstruction of summer temperatures in particular at the low-frequency scale of variability [1-5]. The main concern has generally been the interesting temperature difference between medieval times, Little Ice Age, and the modern period viewing recent and projected warming within the context of natural variability. Less attention is usually paid to the strong multidecadal component of temperature variability in the observational as well as proxy records, which may seriously hamper the identification of an amplified warming signal in the last century in the Arctic and surrounding regions [6]. Some internal climate controls may have influenced regional climate simultaneously with the carbon dioxide induced warming, and Fennoscandian temperature proxies may have recorded both types of potentially coinciding, interacting, or even diverging signals in the decadal-to-centennial scales of variability. Multidecadal variability in Fennoscandian summertime climate may well be related to the AMO (sea surface temperatures (SST)), which has a period of about $40-80$ years, suggested to arise from predictable internal variability of the ocean-atmosphere system $[7,8]$. In addition Arctic air temperature and pressure have been shown to display strong multidecadal variability on similar time scales [9].

The goal here is to study periodicity and trends in growth variability of six recently published and updated millennia length proxies of Scots pine from the northern timberline. If 
the six series show consistent and synchronous interannualto-decadal, decadal-to-multidecadal, as well as centennial and longer types of variability, three versions of each series will be built in order to highlight the three frequency bands separately in these data as well as in various combinations to regional averages (not mixing frequency classes). Growth signals are considered more or less frequency dependent if they all show similar and coherent behavior in the suggested frequency ranges and may thus be usefully combined to regional high-frequency (h-f), medium-frequency (m-f), and low-frequency (l-f) chronologies. Current knowledge is relatively limited regarding spectral details of the l-f and $\mathrm{m}$ $\mathrm{f}$ trends and periodicities in these proxies (except for some older versions of individual proxies for relatively narrow frequency bands $[10,11])$.

All the six series are known predominantly as summer temperature proxies $[1,4,5,12,13]$. This study will provide a synopsis of their rather complex potential as predictors of high-summer (July), standard summer season (JuneAugust), and even some longer warm period temperatures in the three broad frequency ranges. The results will serve as practical guidelines for selecting proper proxy types and choice of indexing methods with respect to required response period as well as frequency band. Such knowledge is crucial in various multiproxy applications (see, e.g., [4]) where the amplitude, duration, and timing of changes are important. In particular, our goal is to reconstruct temperature variability at the multidecadal scale, which would be useful in interregional comparisons of meaningful periodicity in land surface temperatures in the subarctic region as well as between land and sea surface temperatures of larger fields in the search for periodic patterns (both intrinsic and external to the climate system). In order to gain insight into this topic we will here compare the well-known cycles of annual AMO with m-f periodicity in these data. Since Gray et al. [14] successfully reconstructed the AMO using a large network of tree-growth chronologies (including one early version of our data series), it will be interesting to test an AMO model now with these six longer and updated series.

In calibration model performance will be evaluated in each case based on known stringent verification tests. The aim is to provide feasible models in three scales from h-f to l-f with increasing proportion of low frequency variance (where $h-f$ necessarily overlaps in $m-f$ and $m-f$ overlaps in $1-f)$. The linear relationship between tree growth at the three frequency ranges and temperature over the whole range of target variations (unfiltered) is analyzed. It would be reasonable to expect model fit generally to increase if meaningful lower frequencies are added to the pool of predictors (from $h-f$ to l-f). However the situation is generally more complicated as targets are relatively short series as compared to the proxies, and the longest trends in tree growth can only have a partial match in temperature. In an about 100 -year temperature record (usual in calibrations in this region), the detection of verifiable trends is restricted to a maximum of about 50 years (interpolation). However, if a good partial fit of a longer trend is found, some extrapolation is usually reasonable. The results also aim to contribute to the largely missing debate on the uncertainties and even disagreement of regional reconstructions or proxies as compared to the considerable attention over the discrepancies in hemispheric reconstructions [15].

\section{Materials and Methods}

The main interest here is in the inland region, which is occupied by the Fennoscandian Shield and forms a relatively homogeneous peneplane at the modest altitudes of about 200 to $500 \mathrm{~m}$ a.s.l. with sharp climatic and geobotanical boundaries in the east and west [16-20]. Climate in this interior region is arguably more homogeneous and continental without the more marine coastal regions. General location for all six data sets is the northern timberline, between the Swedish Scandes and the Khibiny Low Mountains region.

The data include the recently updated and bias-corrected ring width (SWR) and maximum density (SXD) from Sweden $[5,21,22]$, ring width (FRW), height increment (FHI), and maximum density (FXD) from Finland [4, 23], as well as ring width (RRW) from the Kola peninsula, Russia [4, 24]. The ring width (SRW and FRW) data sets are large, including data from 650 and 536 trees, respectively. The others are smaller, consisting of samples from 167 (FHI) to 78 trees (FXD). Height data (FHI) are shifted by one year for making comparisons possible, because height growth reflects conditions in the previous year $[25,26]$.

Four long, monthly climate records represent the regional temperatures over their common period from 1908 to 2002 [17]. Tornedalen (Sweden) composite record is available as continuous between 1816 and 2002 [27], Karasjok (Norway) record since 1876, Karesuando (Sweden) record since 1890, and Sodankylä (Finland) record since 1908. Tornedalen and Karesuando are the closest to the western (Swedish RW and $\mathrm{XD})$ sampling sites, Karasjok and Sodankylä to the central (Finnish RW, HI, and XD) sites, and Sodankylä is the closest to the eastern (Russian RW) site. Karasjok is the northernmost and Sodankylä and Tornedalen the southernmost climate records. Additional verification is obtained from the Bottenviken compilation series [28], which is based on the data from six stations (Abisko, Karesuando, Kvikkjokk, Jokkmokk, Haparanda, and Piteå, mainly from somewhat more western and southern locations than the ones used in calibrations here) in northern Sweden. Moreover, annual AMO anomalies [29] are used in multidecadal comparisons.

Regional curve standardization (RCS; see [5, 30-32]), 180-year and 30-year splines (see [33-35]) were used in indexing. They are well-known methods in dendroclimatology and frequently applied in targeting various frequencydependent responses in proxy-based reconstructions [36, 37]. The RCS method is expected to preserve any l-f signal up to wavelengths exceeding the lengths of the individual segments used in building the chronologies. On the other hand, the 180-year splines will highlight m-f signal by removing the lowermost frequencies, the most multicenturytimescale variance potentially present in the data. The $30-$ year spline extracts the $\mathrm{h}-\mathrm{f}$ signal and will remove in addition much of the multidecadal and all longer scale variances. The three frequency ranges correspond to interannual-todecadal, interannual-to-multidecadal, and centennial types 
of variability in the time domain. Furthermore, digital lowpass filters were used to extract the chronology variance with a frequency lower than 10 years [38-40].

We used Fourier analysis to define the spectral content in the chronologies, that is, a spectral description in terms of cycles of varying length, the actual frequencies that generate the original series. The wavelet approach has advantages over the more traditional methods for analyzing potentially nonstationary signals, which have discontinuities and nonperiodic characteristics [41-43]. In the complex interactions in climate there are a number of components which tend to damp out the more rapid fluctuations. Thus climate time series with lower frequency/longer period cycles have to contain a greater proportion of the observed variance to achieve the same significance as higher frequency/shorter period features [44]. In the time domain confidence intervals (c.i.) were calculated separately for the $\mathrm{l}-\mathrm{f}$ and $\mathrm{m}-\mathrm{f}$ ranges of growth variability using nonparametric bootstrap method (sampling with replacement) [45, 46]. Arithmetic averages (AA) and weighted averages (WA) (see [47] and references therein) were applied in combining regional chronologies.

Simple linear regression models (transfer functions) [12, 48-50] were developed for each of the three versions of the six chronologies and their averages to be used in turn as climate predictors. Individual models were then tested in split period calibration verification, where the total calibration period (1908-2002) was divided into two equal 48-year halves: 1908-1955 and 1955-2002. These subperiods, used for calibration during one period and verification during the other, are referred to as early calibration-late verification and late calibration-early verification (EC-LV and LC-EV, resp.). Both subperiods should produce positive values of reduction of error (RE) $[12,38,51]$ and coefficient of efficiency (CE) $[12,51]$ statistics for the model in order to pass the verification tests. $\mathrm{RE}$ and $\mathrm{CE}$ values may vary from +1 to $-\infty$, with 0 indicating that the reconstruction model performs no better as a predictor than the calibration (RE) or verification (CE) period mean value. The models are also compared using coefficient of determination $\left(R^{2}\right)$ and explained variance $\left(r^{2}\right)$.

\section{Results}

3.1. Periodicity, Trends, and Interannual Shifts in Growth. Five of the six l-f series (RCS, no filtering) share significant oscillatory mode at 200-250 years of cycle length (Figure 1) and SXD shows even longer-term features (Figure 1(b)). Most series also have strong multidecadal scale variability, viz. significant peaks at 67 years in SRW and SXD, as well as at 83-125 years in FRW, FXD, and RRW. Although all series have cumulated some concentration of variance at $\mathrm{m}-\mathrm{f}$ scale, the two western series (SRW and SXD) have 67-year peaks and both of the XD series have a (possibly related) peak at 33 years. In addition the two XD series differ evidently from the others with still distinctly higher frequency content at $0.1-0.3$ cpa (Figure 1). Based on these results the common spectral content of these data is concentrated principally on the relatively narrow frequency ranges from $0.005 \mathrm{cpa}$ to $0.5 \mathrm{cpa}$; that is, periodicities are from 200 to 2 years.
Next, the six l-f time series were low-pass filtered, normalized, and then averaged (c.i. around the mean in Figure 2(a), in 1006-1996, since six years are lost at both ends due to filtering). Correlations between these $\mathrm{l}$-f series are all positive, ranging from 0.81 (FRW-FHI) to 0.21 (FHI-RRW) with a mean value of 0.52 . Correlation declines in the three RW series with increasing distance; the $r$-value (from west to east) is 0.55 between SRW and FRW, 0.34 between SRW and RRW, and 0.49 between FRW and RRW. The two density series (SXD and FXD) have high linear association $(r=0.66)$. However their correlation is lower than that between either the two western or central RW and XD series (SRW-SXD, $r=0.73$ and FRW-FXD, $r=0.77$ ).

The mean regional l-f chronology (Figure 2(a)) shows an overall increasing trend (regression of growth on time in 1006-1996; $y=0.0006 x$ ), reflecting the diverse longterm rates of change in the original series. Producing a distribution of the mean values and then locating the lower and upper bounds of this distribution, five significant (95\% c.i.) periods of positive growth in the average series are discerned: 1083-1104, 1159-1178, 1428-1436, 1750-1769, and the longest from 1918 to 2002, separated by periods of poor growth of varying lengths and magnitudes between them. These periods are determined in the regional growth signal within the uncertainty limited by (sources of error) interregional differences (e.g., western, central, and eastern provenance) as well as differences due to proxy type (RW, $\mathrm{XD}$. and $\mathrm{HI}$ ). The highest values in the second millennium are clearly recorded in the last century (Figure 2(a)). The bootstrapped confidence intervals are nonsymmetric and particularly wide in the first and last centuries.

The six $m-f$ (filtered) series are more synchronous than the l-f series as their average has narrower c.i. and many more significant periods (Figures 2(a) and 2(b)) in addition to having a higher mean correlation $(r=0.58)$. Correlation is the highest between FRW and FXD $(r=0.74)$ as well as between FRW and FHI $(r=0.74)$ and the lowest between SXD and FHI $(r=0.39)$ as well as between SXD and RRW $(r=0.39)$. Twelve distinct and significant (95\% c.i.) periods of above average growth are dated (Figure 1(b)): 1083-1101, 1157-1183, 1283-1289, 1411-1447, 1489-1498, 1535-1547, 1559$1572,1625-1634,1653-1665,1750-1767,1850-1862$, and the longest in 1920-1956. The confidence limits indicate wider spread in the original six series in the 13th and 14th centuries than in the rest of the $\mathrm{m}-\mathrm{f}$ series.

The average of the six h- $\mathrm{f}$ (no filtering) series represents the $\mathrm{h}-\mathrm{f}$ variability in this work (Figure 2(c)). Average correlation between all the six series is 0.41 . It is the highest between SRW and FRW $(r=0.68)$ and the lowest between SXD and FHI $(r=0.22)$. In the mean series growth was the lowest in 1601 ( -2.6 s.d. units below the mean) and the highest in 1826 (2.4 s.d. units above the mean). The greatest biennial shifts (difference between any two consecutive years) occurred between 1600 and 1601 (3.8 s.d. from 1.2 to -2.6 ) as well as between 1640 and 1641 (3.8 s.d. from 1.8 to -2.0 ). In the 20th century, 1903 was unusually low and 1937 was high in the growth index. It is worth noticing that even this $\mathrm{h}-\mathrm{f}$ series has evident decadal fluctuations. 


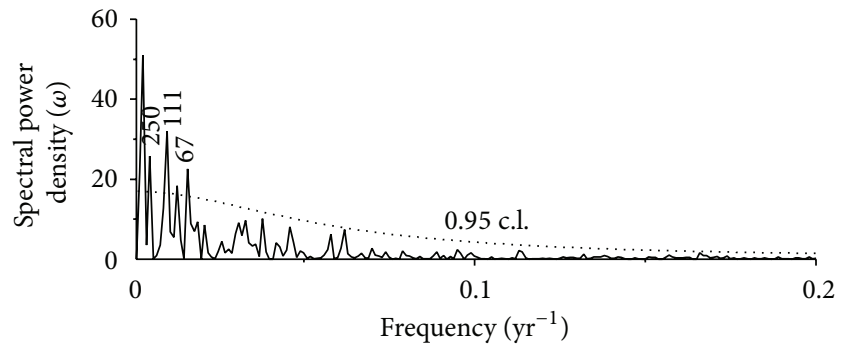

(a)

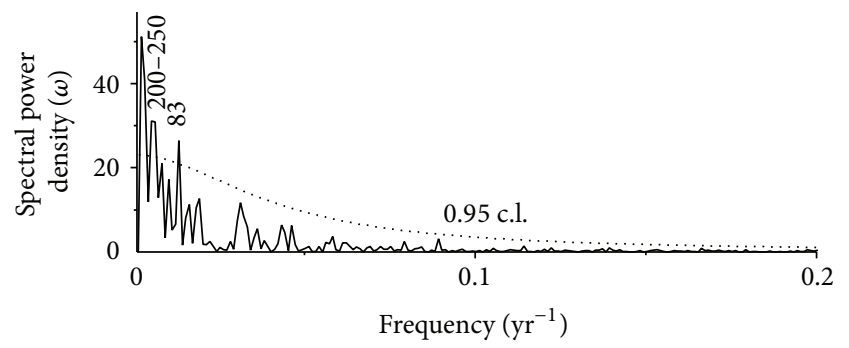

(c)

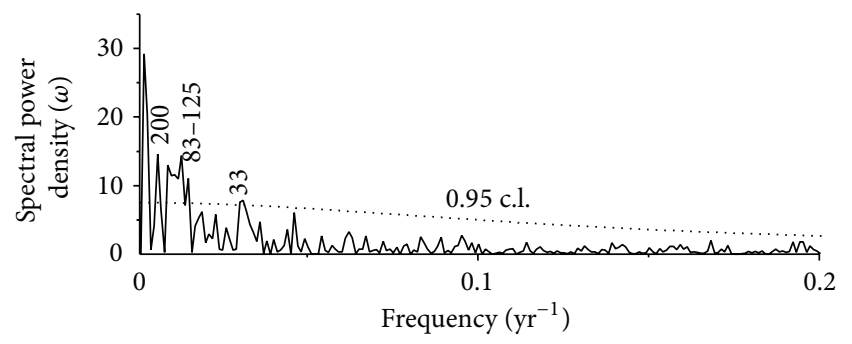

(e)

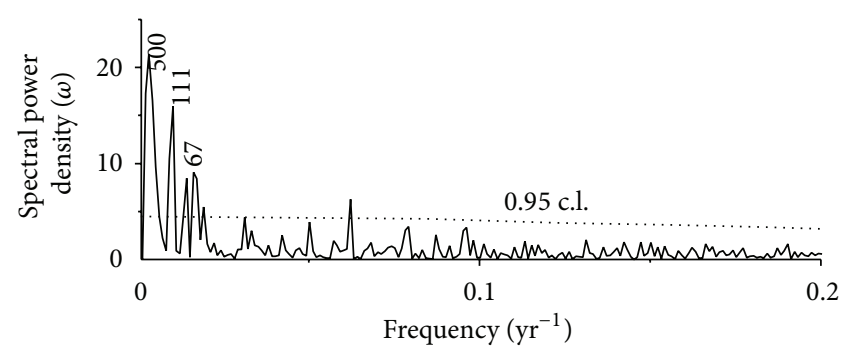

(b)

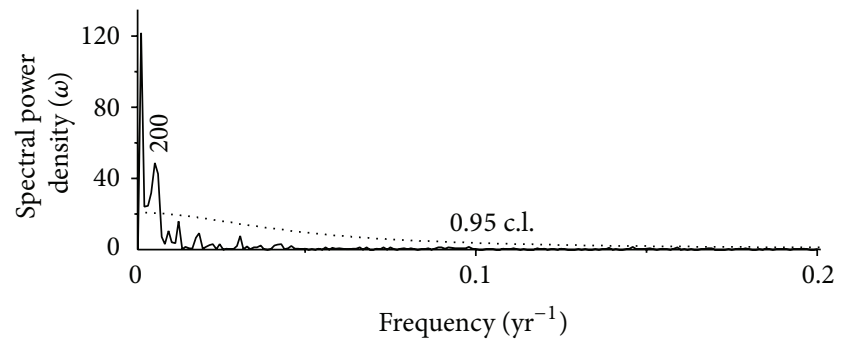

(d)

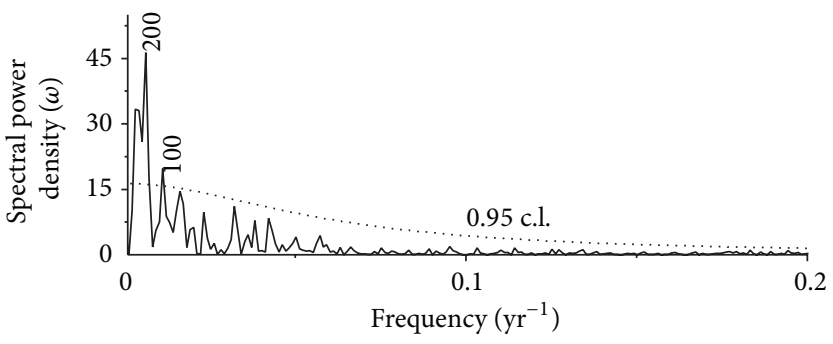

(f)

FIGURE 1: The Fourier spectra of the six growth-based chronologies (RCS indexing) of Scots pine. Smooth lines are 0.95 confidence levels, calculated for red noise with AR(1) coefficients $\alpha$ : (a) SRW ( $\alpha=0.7$ ), (b) SXD ( $\alpha=0.20)$, (c) FRW ( $\alpha=0.77$ ), (d) FHI ( $\alpha=0.75$ ), (e) FXD $(\alpha=0.43)$, and (f) RRW $(\alpha=0.69)$. Cpa is cycles per annum.

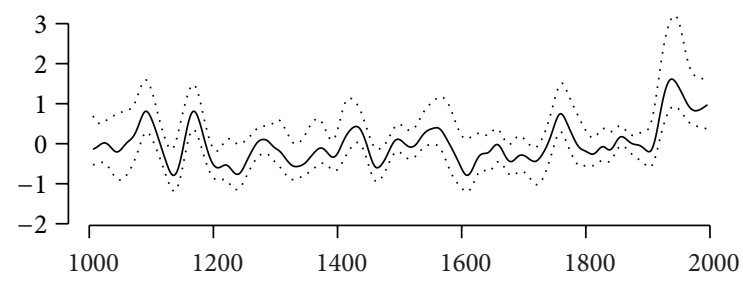

(a)

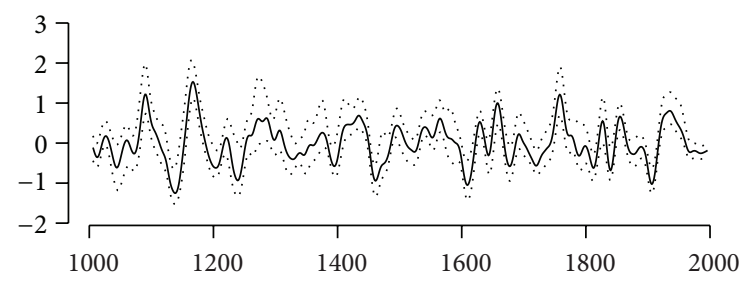

(b)

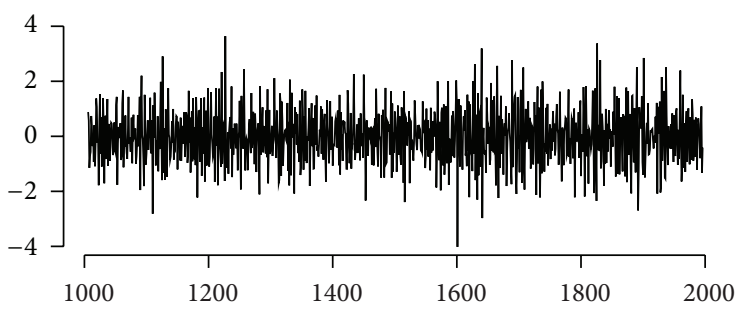

(c)

Figure 2: C.i. for the mean l-f series (RCS, low-pass filtering; (a)), for the $\mathrm{m}$-f series (low-pass filtering of the six 180 -year spline indexed series; (b)) and the average of $\mathrm{h}-\mathrm{f}$ series (30-year splines, without low-pass filtering; (c)). 


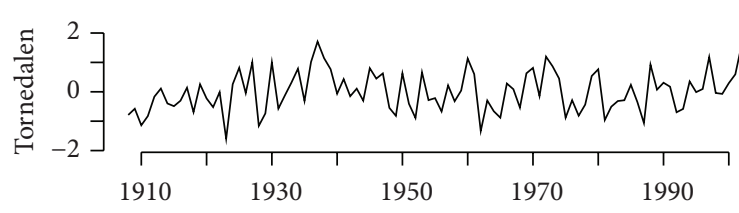

(a)

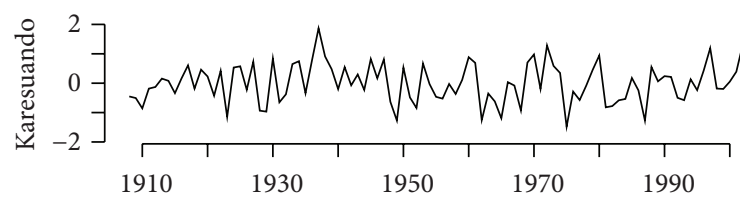

(c)

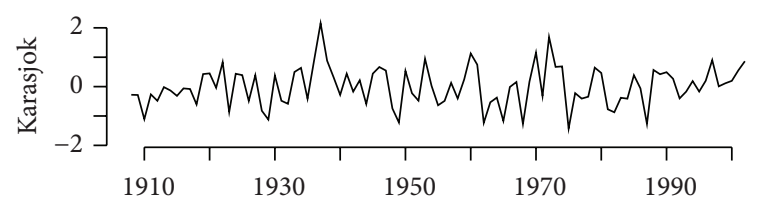

(b)

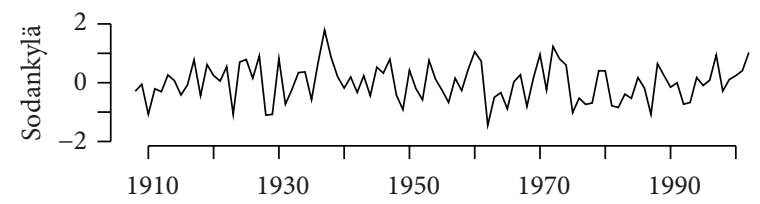

(d)

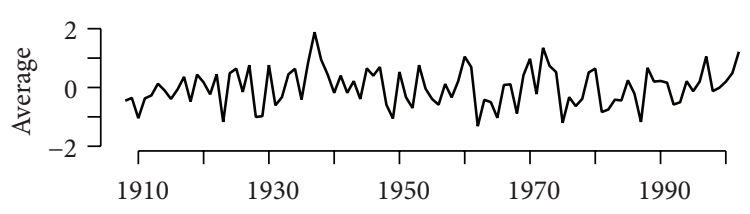

(e)

FIgURE 3: Mean June-August temperatures of the four records and their average (z-scores in 1908-2002).

3.2. Individual Proxies versus July and June-August Temperatures. Our climatic targets are arithmetic averages of normalized monthly data. July temperatures are first modeled using individual proxies and then June-August temperatures using individuals as well as their various combinations. The strength of targeted regional signal is indicated by correlation of June-August mean temperatures among the four stations (Figure 3 ), which vary between 0.88 (TornedalenKarasjok) and 0.94 (Karesuando-Sodankylä), while the mean correlation is 0.92 . This June-August mean has even higher correlation $\left(r=0.96, r^{2}=0.93\right)$ with the June-August mean of the Bottenviken regional temperature record exclusively from northern Sweden [28], used in calibrations in some previous studies [5, 21]. All four records show, for example, cool conditions in the early 20th century until around 1915, the relative warmth of the 1930s, and a recent warming since the late 1980s. Summer temperature (June-August, normalized scale) varies between -1.32 and 1.88 , which here form the limits for interpolation (Figure 3). The mean temperature has a modest positive trend $(y=-0.07+0.0014 x)$ in $1908-2002$.

When the six l-f proxies were individually regressed on mean July temperature (Table $1(\mathrm{~A})$ ), only FXD and RRW produced positive verification statistics. Although SXD has positive RE values in both periods, the CEs are marginally negative. Among the $\mathrm{m}-\mathrm{f}$ series (Table $1(\mathrm{~B})$ ), both of the western series (SRW and SXD) clearly pass the verification tests, while FRW and FHI do not. FXD and RRW series have improved (higher than in l-f) positive RE and CE values. In the higher frequencies (Table $1(\mathrm{C})$ ), in addition to all three RW series, also FHI passes both tests in both periods. However, both SXD and FXD fall just below zero $(-0.02)$ using the more searching CE statistic in the LC-EV period.

The same proxies were then calibrated against mean June-August temperature. In the l-f range (Table 2(A)), SXD, FXD, and RRW pass the tests. Now SXD produces the highest
$\mathrm{RE}$ and $\mathrm{CE}$ values in both periods. In the medium frequencies (as previously in the case of July, Table 1(B)), the four series (SRW, SXD, FXD, and RRW) show positive verification performance. FRW and FHI again have negative RE and $\mathrm{CE}$ values in the $\mathrm{LC}-\mathrm{EV}$ period. In the higher frequencies (Table 2(C)), all six series pass the tests. The three RW series have generally lower explained variance $\left(r^{2} \leq 0.21\right)$ as well as lower RE and CE values $(\geq 0.18)$ than the two other types of proxies (XD and HI), each of which has $r^{2} \geq 0.31$ and both $\mathrm{RE}$ and $\mathrm{CE} \geq 0.28$.

3.3. June-August Temperature Signal in $l-f, m-f$, and $h-f$ Averages. All six series (their $\mathrm{l}-\mathrm{f}, \mathrm{m}-\mathrm{f}$, and $\mathrm{h}-\mathrm{f}$ variants) were averaged to a simple mean (AA) and to a weighted mean (WA) and tested as combined predictors of JuneAugust temperature (Table 3(A)-(C)). Simple mean (AA) produced positive verification results (both RE and CE during both periods) only in the higher frequencies (Table 3(B), Figure 2(c)). The failure of $1-f$ and $m-f$ models here as well as in previous comparisons (Tables 1 and 2) is due to excess growth variability as compared to temperature in the LCEV period, which is most pronounced in FRW and FHI. However, the test values for AA are only slightly weaker than for WA. WA also had positive results in the lowermost frequencies (Table 3(A)). In the medium frequencies, neither method (AA or WA) resulted in an acceptable model (Table 3(B)).

In the next step only those series which individually passed RE and CE tests (in both transfer models for JuneAugust temperatures, Table 2) were weighted and combined, namely, the three l-f series (3RCS; $0.56 *$ SXD + 0.44* FXD $+0.14 * \mathrm{RRW}$ ) and four $\mathrm{m}$-f series (4Spline; $0.22 * \mathrm{SRW}+$ $0.54 * \mathrm{SXD}+0.41 * \mathrm{FXD}+0.16 * \mathrm{RRW})$. When these were calibrated against June-August temperature, both of them passed the verification tests (Table $3(\mathrm{C})$ ). It should be noted 
TABLE 1: Six proxies versus mean July temperatures (average of the four records, no filtering) in 1908-2002. Reduction of error (RE), coefficient of efficiency (CE), and $r^{2}$ during EC-LV (early calibration in 1908-1955 and late verification in 1955-2002) and LC-EV (late calibration in 1955-2002 and early verification in 1908-1955). Only test results with RE and CE $>0$ are included.

\begin{tabular}{|c|c|c|c|c|c|c|}
\hline & \multicolumn{3}{|c|}{ EC-LV } & \multicolumn{3}{|c|}{ LC-EV } \\
\hline & $\mathrm{RE}$ & $\mathrm{CE}$ & $r^{2}$ & $\mathrm{RE}$ & $\mathrm{CE}$ & $r^{2}$ \\
\hline \multicolumn{7}{|c|}{ (A) RCS indexing } \\
\hline FXD & 0.29 & 0.21 & 0.35 & 0.10 & 0.02 & 0.14 \\
\hline RRW & 0.25 & 0.17 & 0.19 & 0.21 & 0.14 & 0.16 \\
\hline \multicolumn{7}{|c|}{ (B) 180-year spline indexing } \\
\hline SRW & 0.30 & 0.22 & 0.29 & 0.24 & 0.18 & 0.32 \\
\hline SXD & 0.26 & 0.18 & 0.25 & 0.15 & 0.08 & 0.12 \\
\hline FXD & 0.35 & 0.28 & 0.36 & 0.14 & 0.07 & 0.15 \\
\hline RRW & 0.27 & 0.19 & 0.21 & 0.23 & 0.17 & 0.18 \\
\hline \multicolumn{7}{|c|}{ (C) 30-year spline indexing } \\
\hline SRW & 0.27 & 0.19 & 0.30 & 0.4 & 0.34 & 0.43 \\
\hline FRW & 0.29 & 0.21 & 0.39 & 0.13 & 0.05 & 0.24 \\
\hline FHI & 0.52 & 0.47 & 0.57 & 0.23 & 0.17 & 0.23 \\
\hline RRW & 0.18 & 0.09 & 0.25 & 0.27 & 0.20 & 0.33 \\
\hline
\end{tabular}

TABLE 2: Six proxies versus mean June-August temperatures in 1908-2002 (no filtering). RE, CE, and $r^{2}$ during EC-LV (1908-1955 and 19552002) and LC-EV (1955-2002 and 1908-1955). Only test results with RE and CE > 0 are included.

\begin{tabular}{|c|c|c|c|c|c|c|}
\hline & \multicolumn{3}{|c|}{ EC-LV } & \multicolumn{3}{|c|}{ LC-EV } \\
\hline & $\mathrm{RE}$ & $\mathrm{CE}$ & $r^{2}$ & $\mathrm{RE}$ & $\mathrm{CE}$ & $r^{2}$ \\
\hline \multicolumn{7}{|c|}{ (A) RCS indexing } \\
\hline SXD & 0.53 & 0.53 & 0.56 & 0.53 & 0.53 & 0.56 \\
\hline FXD & 0.43 & 0.42 & 0.44 & 0.42 & 0.42 & 0.44 \\
\hline RRW & 0.1 & 0.1 & 0.11 & 0.15 & 0.15 & 0.17 \\
\hline \multicolumn{7}{|c|}{ (B) 180-year spline indexing } \\
\hline SRW & 0.17 & 0.17 & 0.21 & 0.07 & 0.07 & 0.28 \\
\hline SXD & 0.47 & 0.47 & 0.54 & 0.45 & 0.45 & 0.57 \\
\hline FXD & 0.35 & 0.35 & 0.44 & 0.27 & 0.26 & 0.43 \\
\hline RRW & 0.12 & 0.12 & 0.13 & 0.15 & 0.15 & 0.18 \\
\hline \multicolumn{7}{|c|}{ (C) 30-year spline indexing } \\
\hline SRW & 0.18 & 0.17 & 0.21 & 0.14 & 0.13 & 0.20 \\
\hline SXD & 0.49 & 0.49 & 0.50 & 0.41 & 0.41 & 0.42 \\
\hline FRW & 0.14 & 0.14 & 0.16 & 0.11 & 0.10 & 0.15 \\
\hline FHI & 0.46 & 0.46 & 0.48 & 0.28 & 0.28 & 0.31 \\
\hline FXD & 0.39 & 0.39 & 0.40 & 0.37 & 0.37 & 0.38 \\
\hline RRW & 0.16 & 0.16 & 0.17 & 0.14 & 0.14 & 0.15 \\
\hline
\end{tabular}

that since sample replication is at least five in 1000-2002 in each of the six series it is thus $>15$ in 3RCS model, $>20$ in 4 Spline model, $>10$ in the XD models, and $>30$ in models where all six series were included.

Because of the persistence in the time series of tree growth they are usually filtered to enhance the signal to be analyzed. It is often recommendable to also reconstruct $1-f$ and $h-f$ variations separately smoothing the proxy and instrumental series prior to calibration $[3,52]$. In order to further study the correspondence between these two mean series (3RCS and 4Spline) and the target above decadal scales, the l-f and $\mathrm{m}$ $\mathrm{f}$ proxies as well as the June-August temperature were lowpass filtered (10-year smoothing; see [38-40]) and then tested again (using correspondingly shorter 42-year calibration and verification periods in 1914-1996 due to filtering). The verifications of these models produced positive results for both; however, here the l-f model was superior to the m-f model.

The four successful multiproxy WA model combinations for June-August temperature (Table 3) were recalibrated using the full 95-year calibration period (Figure 4). The mean of all six RCS-indexed series (with the highest weight on SXD and the lowest on FRW and RRW) produced a slightly inferior model as compared to the 3RCS series $\left(R^{2}=0.57-0.59,3 R C S\right.$ in Figure $\left.4(\mathrm{c})\right)$. In the $\mathrm{m}-\mathrm{f}$ scale the only successful combination (4Spline, Figure 4(b)) has 
TABLE 3: Simple mean (AA) and weighted mean (WA) of all six series versus mean June-August temperatures (no filtering). The WA of RCSbased (A) and 30-year spline indexed series (B) (see also Figure 4(a)). Only test results with RE and CE $>0$ are included. The WAs of those three RCS-indexed series (3RCS; $0.56 * \mathrm{SXD}+0.44 * \mathrm{FXD}+0.14 * \mathrm{RRW})$ and four 180-year spline indexed series (4Spline; $0.22 * \mathrm{SRW}+$ $0.54 * \mathrm{SXD}+0.41 * \mathrm{FXD}+0.16 * \mathrm{RRW}$ ) which individually passed RE and CE tests (see Table 2) versus mean June-August temperatures (C; the full models shown in Figures 4(b) and 4(c)).

\begin{tabular}{|c|c|c|c|c|c|c|}
\hline & \multicolumn{3}{|c|}{ EC-LV } & \multicolumn{3}{|c|}{ LC-EV } \\
\hline & $\mathrm{RE}$ & $\mathrm{CE}$ & $r^{2}$ & $\mathrm{RE}$ & $\mathrm{CE}$ & $r^{2}$ \\
\hline \multicolumn{7}{|l|}{ (A) RCS } \\
\hline WA & 0.55 & 0.55 & 0.67 & 0.21 & 0.21 & 0.53 \\
\hline \multicolumn{7}{|c|}{ (B) 30-year spline indexed } \\
\hline AA & 0.56 & 0.56 & 0.57 & 0.50 & 0.50 & 0.51 \\
\hline WA & 0.65 & 0.65 & 0.65 & 0.58 & 0.57 & 0.58 \\
\hline \multicolumn{7}{|c|}{$\begin{array}{l}\text { (C) WAs of selected three RCS and four } \\
180 \text {-year spline indexed series }\end{array}$} \\
\hline 3RCS & 0.58 & 0.58 & 0.61 & 0.53 & 0.52 & 0.58 \\
\hline 4Spline & 0.46 & 0.46 & 0.60 & 0.28 & 0.28 & 0.60 \\
\hline
\end{tabular}

intermediate values between $\mathrm{l}-\mathrm{f}$ and $\mathrm{h}-\mathrm{f}$ scales. The WA of all six h-f series (30-year spline indexed, Figure 4(a)) shows the best fit with the target $\left(R^{2}=0.62\right)$. Here the modeled and observed values have particularly synchronous (one- or two-year) peaks in, for example, 1923, 1928-1929, 1949, 1962, and 1979-1980 (Figure 4(c)). The l-f model reproduces, for example, the longer increasing trend from 1908 to 1937 as well as that of the last fifteen years more consistently than the $\mathrm{h}-\mathrm{f}$ series (Figures 4(a)-4(c)). However, the reproduction of the twin peaks in 1969-1974 is noticeably poorer.

The two density series (SXD and FXD) were further combined (WA) in both the $1-f$ and $m-f$ ranges and calibrated against longer warm season periods (Table 4). Three different periods were used, four months mean temperature from May to August (A), five months mean from April to August (B), and six months mean from April to September (C). All these six proxy combinations (both l-f and $m-f$ ) pass the verification tests (RE and CE in both periods: EC-LV and LC-EV) for all three temperature periods. Model fit $\left(R^{2}\right)$ recalibrated using all available data varies from 0.48 to 0.58 . The weights in WA are rather equal for SXD and FXD, ranging from 0.51 to 0.34 , but slightly favoring SXD. The best empirical models (for May-August temperatures, Table 4) for the $\mathrm{l}-\mathrm{f}$ and $\mathrm{m}$ - $\mathrm{f}$ bandwidths of the two XD-series were derived by recalibration in 1908-2002 with $R^{2}=0.58$ for the l-f and $R^{2}=0.53$ for the $\mathrm{m}-\mathrm{f}$ model.

3.4. Building a Proxy for Subcentury Scale Temperature Variability. A composite $\mathrm{m}-\mathrm{f}$ summer temperature proxy was produced using the 4Spline variant (WA of SRW, SXD, FXD, and RRW) (Figure 5(a), calibration in Table 3(C) and Figure 4(b)). Several significant (above 0.99 c.l.) multidecadal characteristics from periods of 33 and 67 years up to a peak exceeding a century (111 years) are highly significant in the Fourier spectrum (Figure 5(c)). The wavelet spectrum shows that the fluctuations spreading over these bandwidths are particularly apparent in the 12th and 17th centuries. On the other hand there is a noticeable lack of significant features residing in the decadal to bidecadal ranges (Figures 5(b) and 5(c)). In this multidecadal scale the 20th century does not stand out as unusual in the past millennium.

In order to assess the correspondence of amplitude, duration, and timing of recent cycles between Fennoscandia and the North Atlantic the $\mathrm{m}$-f proxy (4Spline based) and AMO were also visually compared (Figure 6). Correlation between the two series (in 1866-1992) is 0.27 , rising to 0.49 using 10 year moving averages (MA, Figure 6(a)) and to 0.56 using 20year MA. Presuming Fennoscandian proxy variability is preceding the AMO cycle (evident in Figure 6(a)) and correcting for (removing) this 10-year time shift the correlation rises to $0.34,0.73$, and 0.82 , respectively. The 20 -year MA effectively removes all higher frequencies and the relationship seems in agreement particularly during early to mid-20th century. This illustrates that multidecadal variability in Fennoscandian and North Atlantic climates is closely similar in scale and length.

To assess the potential of these data in modelling the AMO (e.g., time stability of such statistical relationship), all six $\mathrm{m}$-f proxies were also compared to annual SST anomalies. Screening the pool of candidate predictors it was also possible to build a transfer model for the AMO. The WA of three series (SRW, FXD, and RRW) was used with the same calibration (1922-1990) as well as verification (1856-1921) periods as in Gray et al. [14]. This model passed the verification trials (RE $=0.05$ and $\mathrm{CE}=0.05$ ), but model performance is the lowest $\left(R^{2}=0.17\right)$ in this work.

\section{Discussion}

Five of the six series from different parts of northern Fennoscandia show evidence of a dominant 200-250 years of periodicity. Similar periodicity was found significant in a harmonic decomposition of the average of six central European instrumental as well as stalagmite proxy series from the Austrian Alps in 500-1935 [53]. However, the pronounced minimum during recent centuries appears in our proxies somewhat later (in the early 20th century) as compared to the 1880s noted by Lüdecke et al. [53]. Previously Helama et 


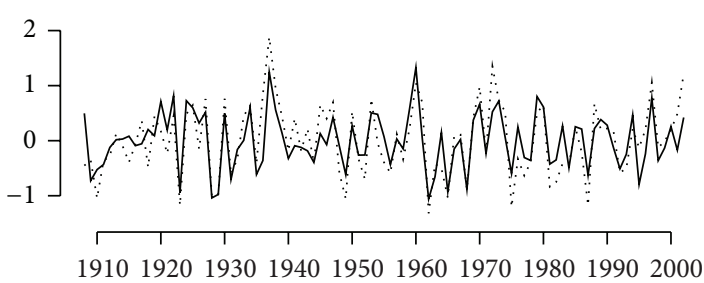

(a) High-frequency model; all six $R^{2}=0.62$

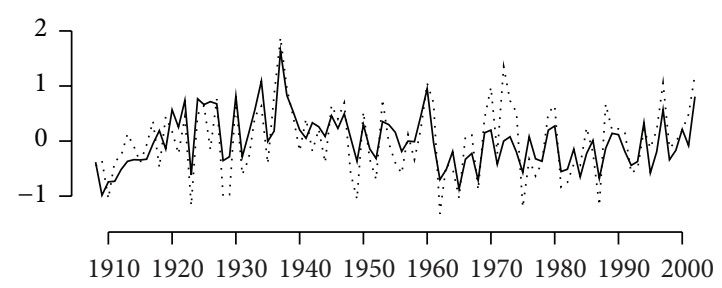

(b) Medium-frequency model; 4 Spline $R^{2}=0.56$

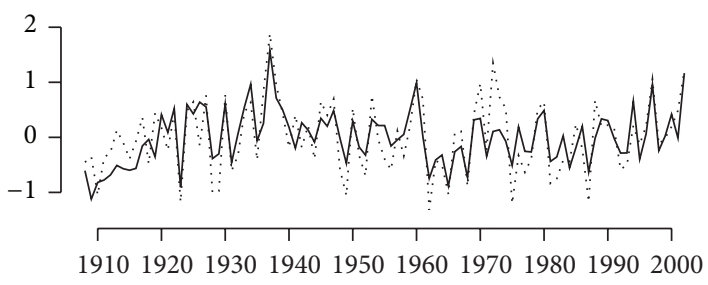

(c) Low-frequency model; 3RCS $R^{2}=0.59$ (all six model $R^{2}=$ $0.57)$

FIGURE 4: Final linear regression models on June-August mean as target (dotted line) with various combinations of proxies recalibrated in 1908-2002 (solid line): (a) WA of all six h-f (30-year spline indexed) series, (b) WA of SRW, SXD, FXD, and RRW (four 180-year spline indexed, 4Spline) $\mathrm{m}-\mathrm{f}$ series, and (c) WA of the SXD, FXD, and RRW (3RCS series).

TABLE 4: Weighted averages of the Swedish and Finnish density series (SXD and FXD) built using two types of indexing (RCS and 180-year splines, Sp) versus a four-month mean temperature (no filtering) from May to August (A), a five-month mean from April to August (B), and a six-month mean from April to September (C). Only test results with RE and CE $>0$ are included.

\begin{tabular}{lccccrr}
\hline & & EC-LV & & \multicolumn{2}{c}{ LC-EV } \\
& RE & CE & $r^{2}$ & RE & CE & 0.55 \\
\hline RCS_A & 0.55 & 0.54 & 0.56 & 0.55 & 0.57 \\
Sp_A & 0.33 & 0.33 & 0.55 & 0.29 & 0.28 \\
RCS_B & 0.49 & 0.48 & 0.50 & 0.49 & 0.48 & 0.58 \\
Sp_B & 0.26 & 0.25 & 0.47 & 0.53 & 0.51 \\
RCS_C & 0.44 & 0.43 & 0.45 & 0.32 & 0.52 \\
Sp_C & 0.24 & 0.24 & 0.41 & 0.52 & 0.53 \\
\hline
\end{tabular}

Weights used in averaging: $0.51 * \mathrm{SXD}+0.47 * \mathrm{FXD}\left(\mathrm{RCS} \_\mathrm{A}\right) ; 0.47 * \mathrm{SXD}+0.42 * \mathrm{FXD}\left(\mathrm{SP} \_\mathrm{A}\right) ; 0.47 * \mathrm{SXD}+0.41 * \mathrm{FXD}\left(\mathrm{RCS} \_\mathrm{B}\right) ; 0.42 * \mathrm{SXD}+0.35 *$ FXD (Sp_B); $0.46 *$ SXD + $0.39 *$ FXD (RCS_C); $0.41 *$ SXD + $0.34 *$ FXD (Sp_C).

al. [54] discussed centennial and multidecadal temperature variability over Northern Fennoscandia that bears a potential link to oceanic origins $[8,55]$ and reviewed the paleoclimatic literature relevant to the topic.

Several individual Fennoscandian tree growth-based proxies have indicated prominent spectral features at about 23,30 , and 90 years, $23-33$ years, and $30.8-31.8$ and $80.3-87.7$ years in Finland $[11,25,56]$ as well as relatively time-stable peaks at 32-33 years and at $\sim 55-100$ years in Sweden [10]. In an analysis of seven northern hemisphere temperature reconstructions (including, e.g., [15, 57-59]) Ogurtsov et al. [60] reported that they have an unambiguous 60-80-year multidecadal variability in common (AD 1000-1930), which is close to the range of a 67-year cycle indicated by our data. The 4 Spline reconstruction without the more or less discrepant secular characteristics (see [17]) clearly records a bimodal structure-c.a. 110-year and 60-70-year variationsas well as a 33-year climatic cycle (similar to the Bruckner cycle). Based on these evidence there exists obvious potential for analyses in subcentury scales and a real possibility to gain insight into the extent of general natural variability in the subarctic region.

In the lower frequencies of these data (low-pass filtering of the RCS and 180-year spline indexed series) several growth surges and troughs coincide in each group and they were also dated in the averages in the time domain (statistically significant fluctuations; five in $1-f$ and 12 in $\mathrm{m}$-f range). The latest, 37-year period (1920-1956) is the longest continuous multidecadal scale surge and the 85-year period (1918-2002) is the longest l-f surge. The combined m-f chronology is evidently more consistent than the l-f chronology as the more diverging $1-f$ trends are left out. Overall signal strength (measured as mean correlation) between the six series is higher in $\mathrm{m}-\mathrm{f}(r=0.58)$ than either in l-f $(r=0.52)$ or in h-f series $(r=0.41)$. At least part of the l-f disagreement is possibly due to the (noisy) RCS method, which is usually recommended for large data sets of various age classes of trees for each year, ideally grown under a range of representative ecological conditions [10, 21, 61-64]. Even the ample data bases of SRW and FRW may not fully meet these requirements. 


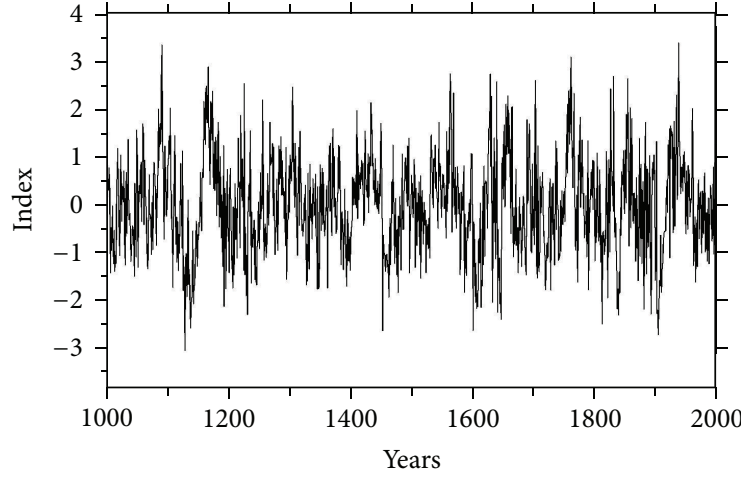

(a)

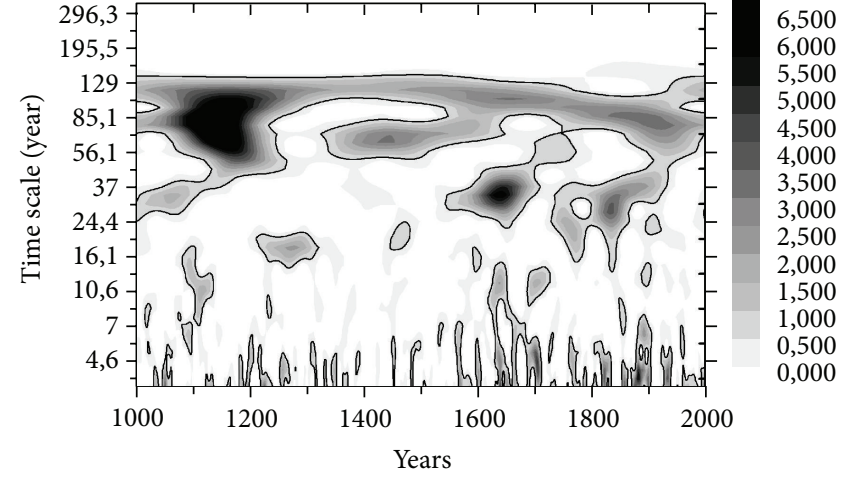

(b)

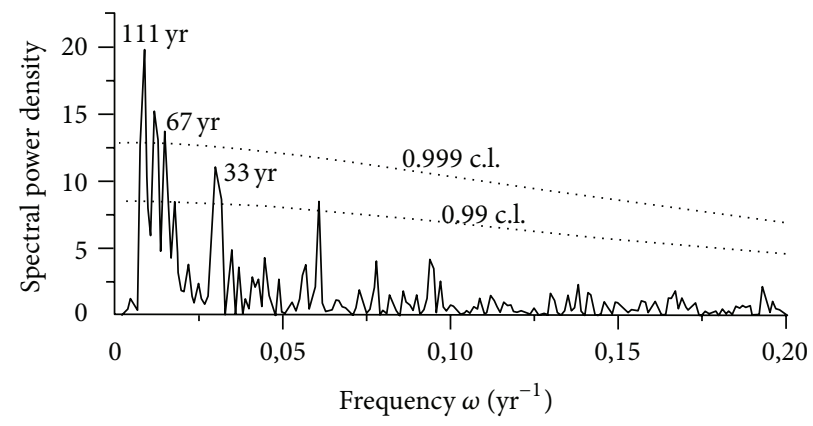

(c)

FIGURE 5: The m-f proxy of summer temperatures since AD 1000 ((a), unfiltered). Wavelet spectrum (b) and Fourier spectrum (c) of this time series.

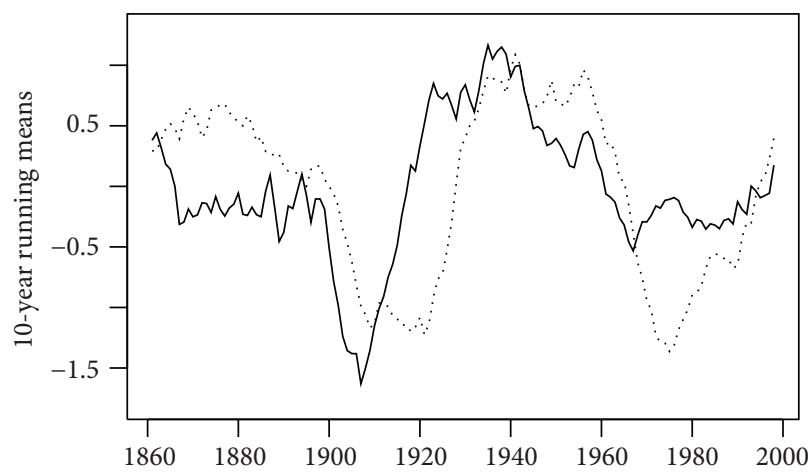

(a)

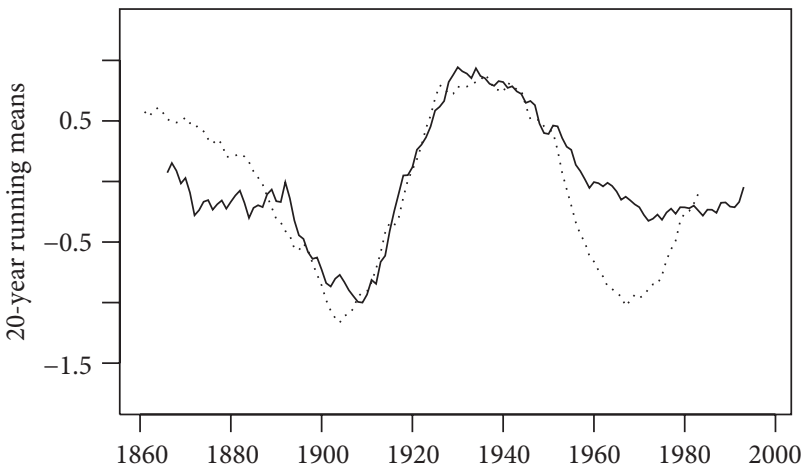

(b)

Figure 6: Comparison of annual AMO cycle (dash) and the Fennoscandian multidecadal ( $\mathrm{m}-\mathrm{f}$ ) summer temperature proxy (solid) during common period using 10-year (a) and 20-year (b) smoothing.

The four instrumental temperature records used in calibrations show remarkable agreement justifying their averaging to a regional mean. July is usually one of the most important factors among monthly temperatures related to various growth parameters from different parts of the region $[12,21,23,24,54]$. When the six proxies were individually regressed on mean July temperature, only FXD and RRW passed the verification trials in the l-f range, SRW and SXD in the $\mathrm{m}-\mathrm{f}$ range, and all but the two $\mathrm{XD}$ series in the $\mathrm{h}-\mathrm{f}$ range.
The growth response of June-August temperatures has often been found time stable and this period has recently been used in temperature reconstructions in the region $[2-5,17]$. A simple mean of all six proxy series (AA) works as a verifiable predictor of mean June-August temperature only in the hf range (30-year spline indexing, Figure 2(c)) in these data. Although all series pass the tests, the SXD, FHI, and FXD have superior individual calibration and verification performance in this context. This is consistent with the results obtained 
previously by Lindholm et al. [17]. The SXD is obviously the best individual temperature proxy in these three frequency classes, usually most heavily weighted in the various WAs. Otherwise the mean is generally a more powerful predictor of regional temperatures than the individuals. The WA of the six series proved to be a successful predictor of the standard summer period also in the l-f range. Interestingly McCarroll et al. [4] were able to reconstruct June-August temperatures using the simple mean of part of these same data (SRW, SXD, and RRW) in a larger network of nine series. The contrast is plausibly explained mainly by their successful application of different mixed indexing methods (a combination of RCS and splines) as well as inclusion of the coastal areas in both the proxy and climate data and perhaps to a lesser extent by different climate station data as target and different calibration and verification periods.

The use of only a more limited number of $1-f$ and $m-$ f series (those which individually passed the RE and CE tests) produced the best reconstruction models of JuneAugust temperatures. Both types of models (3RCS for l-f and 4Spline for $\mathrm{m}-\mathrm{f}$ ) use (the RW and XD) data from all the three subregions (western, central, and eastern). If the number of predictor variables is further limited to XD series only, it is possible to develop excellent models also for longer summer periods, up to half a year from April to September. Previously this XD quality was associated only with SXD $[10,13,21]$ but now we have successfully combined both SXD and FXD for this purpose, since the FXD clearly shares this wide temperature-response time-window property.

The arithmetic averages of $1-f$ and $m-f$ series show some divergence between low-frequency growth variability and mean regional summer temperatures (especially FRW and FHI), leading to overestimations in calibrations (and consequently poor verification) as seen, for example, in Table 2(A)-(B). The phenomenon is often related to the autocorrelation structure of the series and factored out by smoothing (using weighted moving averages), including predictors from years lagging and/or leading current year of growth. Such model structures were compared by Briffa et al. [12], and various combinations of them have frequently been applied in reconstructions including RW in the region $[13,21,54,65]$. In this work we only compared current year's growth with current temperature. Thus, the rather modest performance of FRW, FHI, and SRW in models of July and June-August temperatures is perhaps not so surprising. The apparently narrow seasonal response pattern of RW series (as compared to broader response time window in XD) generally corresponds with previous results [12, 13, 21, 22, 54, 63].

The autocorrelation structure of a chronology varies through time and depends on the way in which the series have been standardized. Thus, comparisons of indexing methods targeting different (dependent) frequency response are pertinent in dendroclimatological reconstruction work $[16,24,61]$. When the three types (l-f, m-f, and h-f) of reconstruction models are plotted together with the target June-August temperature their differences are not immediately apparent, even between the two extremes-30-year spline and RCS-based series-although the differences in closer detail are distinct and the $\mathrm{h}-\mathrm{f}$ type reproduces the finer details more meticulously than the two other types. This is because their differences lie in the only gradually changing l-f characteristics, long-term trends, and periodicities and calibration is necessarily done in a short period.

Comparison of Fennoscandian summer temperature proxy and annual AMO revealed that although the timing of the periods does not match, the amplitude and duration of the cycles do (in roughly 1880-1950). Furthermore Fennoscandian proxy variability was found preceding the SST cycle by about 10 years. Despite the shift in dating, data from all three subregions (SRW, FXD, and RRW) enabled us to build a model for the AMO. The calibration and verification periods were applied according to Gray et al. [14] in their reconstruction of the AMO using a network of 12 proxies including an early version of the FRW ( $\mathrm{RE}=0.25$ in this model as compared to our model with $\mathrm{RE}=0.05$ ). The 10 -year temporal shift plausibly explains the rather low test values of our AMO model. The comparison also illustrates that the 180year splines (m-f) are a proper method to capture the relevant frequency range in these data.

As AMO is lagging behind it is obviously not a direct cause of multidecadal oscillation in Fennoscandia. However, the similarities between AMO and proxy may well be related to common origin judged purely by arising periodicities. Such widespread multidecadal temperature variability has been reported from the North Atlantic, the Arctic, and in Europe $[9,53,55]$. In Fennoscandian proxies the recognition of such cycles would help to explain part of the uncertainties in the 20th century trends. Soon after the severe drop at the turn of the (19th and 20th) centuries the highest relative growth (and thus pronounced warming) is usually recorded in 1930s-1940s with a peak in 1937 [2, 4], followed by medium values in 1950s-1970s and a recovery only in the last decades. If the 20th century warming occurred already by midcentury this is somewhat in contrast with the gradual warming expectation as a response to rising $\mathrm{CO}_{2}$ concentration in the atmosphere but corresponds to some similarities in SST and Arctic surface air temperature fluctuations, which show two maxima, in 1930s-1940s and in the last decades [9].

Our $\mathrm{m}$-f reconstruction provides a useful tool for interregional comparisons targeting the subcentury scales. As far as climatic signals can be extracted from more or less noisy proxies it is within reason to pay attention to the targeted frequencies in building the proxies. This will also help to understand the challenge in distinguishing between internally driven ocean-atmosphere-land dynamics and externally forced warming signal. Realizing a dominant multidecadal phenomenon also in Fennoscandian climate helps to explain in this work the higher linear association in $m-f$ than either in l-f or h-f growth variability, although the RCSbased model has advantages over the 180-year splines based model, for example, in the decadal-to-centennial scales as was shown by calibration using separate filtered data sets. It may well also in part explain the rather modest model performance of FRW and FHI in the early 20th century due to excess variance in growth as compared to temperature (LC-EV period divergence). Moreover this frequency band easily relates to interregional (statistical) connection between 
Fennoscandian and Arctic climates in particular related to the North Atlantic.

\section{Conclusion}

Six growth-based proxies of Scots pine from the northern Fennoscandian timberline between the Swedish Scandes and the Khibiny Low Mountains were combined to enhance their frequency-dependent properties in growth variability in AD 1000-2002. Five significant periods of good growth were found in the low frequencies and 12 periods in the medium frequencies. Most of the l-f series share significant oscillatory modes in 200-250 years of cycle length and strong multidecadal components. Thus we were able to build a separate $\mathrm{m}-\mathrm{f}$ reconstruction using the 4 Spline model. This series lacks the multicentury frequency bands dominant in the l-f series but has high concentrations of variance with a bimodal structure (ca. 110-year and 60-70-year variations) as well as a 33-year cycle.

Linear transfer models were built for short and long warm seasons using the six proxies in three frequency ranges. When they were individually regressed on mean July temperature only FXD and RRW passed the verification trials in the l-f range, SRW and SXD in the $\mathrm{m}-\mathrm{f}$ range, and all but the two XD series in the h-f range. A simple mean of all six series works as a successful predictor of mean June-August temperature only in the h-f range. The SXD, FXD, and FHI were superior to the RW. On the other hand, the weighted mean of these same six series provides a good model of the standard summer period also in the l-f range. The use of only a more limited number of $\mathrm{l}-\mathrm{f}$ and $\mathrm{m}-\mathrm{f}$ series produced the best reconstruction models of June-August temperatures $\left(R^{2}=0.56-0.62\right)$. Both types of models (three of the RCS series for l-f and four of the 180-year spline indexed series for $\mathrm{m}-\mathrm{f}$ ) use the RW and $\mathrm{XD}$ data from all the three subregions, western, central, and eastern. If predictor variables are further limited only to XD series, it is possible to develop excellent models also for longer summer periods, up to half a year from April to September $\left(R^{2}=0.48-0.58\right)$. Previously this XD quality was associated only with SXD.

We can explain the otherwise peculiar general view of the 20th century by presuming that the growth surge and corresponding warming in the early half of the 20th century are part of a widespread dominant cycle taking place along a rising centennial trend (both types are evident in the l-f and $\mathrm{m}$-f series). It is within reason to link the early multidecadal features more closely to natural phenomena and the overall 20th century rise to external forcing. Analyzing different frequency ranges in proxies will potentially help in meeting a major future challenge to separate the externally forced from internally driven variations (both natural and external components integrated in long records) for the detection and attribution of anthropogenic climate change.

\section{Conflict of Interests}

The authors declare that there is no conflict of interests regarding the publication of this paper.

\section{Acknowledgments}

This work was supported by the Academy of Finland (Grant no. SA 138937). M. G. Ogurtsov expresses his thanks to the exchange program between the Russian and Finnish Academies (Project no. 16), to the program of the Presidium of RAS no. 22, and to RFBR Grants 11-02-00755, 13-02-00277, and 13-02-00783 for financial support.

\section{References}

[1] K. R. Briffa, V. V. Shishov, T. M. Melvin et al., "Trends in recent temperature and radial tree growth spanning 2000 years across northwest Eurasia," Philosophical Transactions of the Royal Society B: Biological Sciences, vol. 363, no. 1501, pp. 22692282, 2008.

[2] U. Büntgen, C. C. Raible, D. Frank et al., "Causes and consequences of past and projected Scandinavian summer temperatures, 500-2100 AD," PLoS ONE, vol. 6, no. 9, Article ID e25133, 2011.

[3] J. Esper, U. Büntgen, M. Timonen, and D. C. Frank, "Variability and extremes of northern Scandinavian summer temperatures over the past two millennia," Global and Planetary Change, vol. 88-89, pp. 1-9, 2012.

[4] D. McCarroll, N. Loader, R. Jalkanen et al., "A, 1200-year multiproxy record of tree growth and summer temperature at the northern pine forest limit of Europe," Holocene, vol. 23, pp. 471484, 2013.

[5] T. M. Melvin, H. Grudd, and K. R. Briffa, "Potential bias in "updating" tree-ring chronologies using regional curve standardisation: re-processing 1500 years of Torneträsk density and ring-width data," Holocene, vol. 23, pp. 364-373, 2013.

[6] I. V. Polyakov, G. V. Alekseev, R. V. Bekryaev et al., "Observationally based assessment of polar amplification of global warming," Geophysical Research Letters, vol. 29, no. 18, pp. 25$1-25-4,2002$.

[7] R. A. Kerr, "A North Atlantic climate pacemaker for the centuries," Science, vol. 288, no. 5473, pp. 1984-1986, 2000.

[8] M. E. Schlesinger and N. Ramankutty, "An oscillation in the global climate system of period 65-70 years," Nature, vol. 367, no. 6465, pp. 723-726, 1994.

[9] I. V. Polyakov, R. V. Bekryaev, G. V. Alekseev et al., "Variability and trends of air temperature and pressure in the maritime arctic, 1875-2000," Journal of Climatology, vol. 16, pp. 20672077, 2003.

[10] K. R. Briffa, P. D. Jones, T. S. Bartholin et al., "Fennoscandian summers from ad 500: temperature changes on short and long timescales," Climate Dynamics, vol. 7, no. 3, pp. 111-119, 1992.

[11] H. H. Lamb, Climate-Present, Past and Future, Methuen, London, UK, 1992.

[12] K. R. Briffa, P. D. Jones, J. R. Pilcher, and M. K. Hughes, "Reconstructing summer temperatures in northern Fennoscandinavia back to AD 1700 using tree-ring data from Scots pine," Arctic \& Alpine Research, vol. 20, no. 4, pp. 385-394, 1988.

[13] K. R. Briffa, T. S. Bartholin, D. Eckstein et al., "A 1,400-year treering record of summer temperatures in Fennoscandia," Nature, vol. 346, no. 6283, pp. 434-439, 1990.

[14] S. T. Gray, L. J. Graumlich, J. L. Betancourt, and G. T. Pederson, "A tree-ring based reconstruction of the Atlantic Multidecadal 
Oscillation since 1567 A.D," Geophysical Research Letters, vol. 31, no. 12, 2004.

[15] J. Esper, E. R. Cook, and F. H. Schweingruber, "Low-frequency signals in long tree-ring chronologies for reconstructing past temperature variability," Science, vol. 295, no. 5563, pp. 22502253, 2002.

[16] M. Lindholm, Reconstruction of past climate from ring-width chronologies of Scots pine (Pinus sylvestris L.) at the northern forest limit in Fennoscandia [Dissertation], University of Joensuu, 1996.

[17] M. Lindholm, T. Aalto, H. Grudd, D. McCarroll, M. Ogurtsov, and R. Jalkanen, "Common temperature signal in four wellreplicated tree growth series from northern Fennoscandia," Journal of Quaternary Science, vol. 27, pp. 828-834, 2012.

[18] M. Lindström, "Northernmost Scandinavia in the geological perspective," Ecological Bulletin, vol. 38, pp. 17-37, 1987.

[19] T. Ahti, L. Hämet-Ahti, and J. Jalas, "Vegetation zones and their sections in northwestern Europe," Annles Botanici Fennici, vol. 5, pp. 169-211, 1968.

[20] S. Tuhkanen, "A circumboreal system of climatic- phytogeographical regions," Acta Botanica Fennica, vol. 127, pp. 1-50, 1984.

[21] H. Grudd, "Torneträsk tree-ring width and density ad 5002004: a test of climatic sensitivity and a new 1500-year reconstruction of north Fennoscandian summers," Climate Dynamics, vol. 31, no. 7-8, pp. 843-857, 2008.

[22] H. Grudd, K. R. Briffa, W. Karlén, T. S. Bartholin, P. D. Jones, and B. Kromer, "A 7400-year tree-ring chronology in northern Swedish Lapland: natural climatic variability expressed on annual to millennial timescales," Holocene, vol. 12, no. 6, pp. 657-665, 2002.

[23] M. Lindholm and R. Jalkanen, "Subcentury scale variability in height-increment and tree-ring width chronologies of Scots pine since AD 745 in northern Fennoscandia," Holocene, vol. 22, no. 5, pp. 571-577, 2012.

[24] Y. M. Kononov, M. Friedrich, and T. Boettger, "Regional summer temperature reconstruction in the Khibiny Low Mountains (Kola Peninsula, NW Russia) by means of tree-ring width during the last four centuries," Arctic and Alpine Research, vol. 41, pp. 460-468, 2009.

[25] M. Lindholm, R. Jalkanen, H. Salminen, T. Aalto, and M. Ogurtsov, "The height-increment record of summer temperature extended over the last millennium in fennoscandia," Holocene, vol. 21, no. 2, pp. 319-326, 2011.

[26] M. Lindholm, M. Ogurtsov, T. Aalto, R. Jalkanen, and H. Salminen, "A summer temperature proxy from height increment of Scots pine since 1561 at the northern timberline in Fennoscandia," Holocene, vol. 19, no. 8, pp. 1131-1138, 2009.

[27] P. Klingbjer and A. Moberg, "A composite monthly temperature record from Tornedalen in northernn Sweden, 1802-2002," International Journal of Climatology, vol. 23, no. 12, pp. 14651494, 2003.

[28] H. Alexandersson, "Temperature and precipitation in Sweden 1860-2001," SMHI Meteorologi, vol. 104, 2002.

[29] A. Kaplan, M. A. Cane, Y. Kushnir, A. C. Clement, M. B. Blumenthal, and B. Rajagopalan, "Analyses of global sea surface temperature 1856-1991," Journal of Geophysical Research C: Oceans, vol. 103, no. 9, pp. 18567-18589, 1998.
[30] K. R. Briffa, P. D. Jones, F. H. Schweingruber, S. G. Shiyatov, and E. R. Cook, "Unusual twentieth-century summer warmth in a 1,000-year temperature record from Siberia," Nature, vol. 376, no. 6536, pp. 156-159, 1995.

[31] K. R. Briffa and F. H. Schweingruber, "Recent dendroclimatic evidence of northern and central European summer temperatures," in Climate Since A.D., 1500, R. S. Bradley and P. D. Jones, Eds., pp. 366-392, Routledge, London, UK, 1992.

[32] E. R. Cook, B. M. Buckley, R. D. D’Arrigo, and M. J. Peterson, "Warm-season temperatures since $1600 \mathrm{BC}$ reconstructed from Tasmanian tree rings and their relationship to large-scale sea surface temperature anomalies," Climate Dynamics, vol. 16, no. 2-3, pp. 79-91, 2000.

[33] E. R. Cook and K. Peters, "The smoothing spline: a new approach to standardizing forest interior tree-ring series for dendroclimatic studies," Tree-Ring Bulletin, vol. 41, pp. 45-53, 1981.

[34] E. R. Cook, A time series analysis approach to tree-ring standardization [Dissertation], University of Arizona, 1985.

[35] E. R. Cook, K. R. Briffa, S. Shiyatov, and V. Mazepa, “Tree-ring standardization and growth-trend estimation," in Methods of Dendrochronology: Applications in the Environmental Science, E. R. Cook and L. Kairiukstis, Eds., pp. 104-122, Kluwer Academic, Dordrecht, The Netherlands, 1990.

[36] V. Trouet, J. Esper, N. E. Graham, A. Baker, J. D. Scourse, and D. C. Frank, "Persistent positive north atlantic oscillation mode dominated the medieval climate anomaly," Science, vol. 324, no. 5923, pp. 78-80, 2009.

[37] H. F. Zhu, X. Q. Fang, X. M. Shao, and Z. Y. Yin, "Tree ringbased February-April temperature reconstruction for Changbai Mountain in Northeast China and its implication for East Asian winter monsoon," Climate of the Past, vol. 5, no. 4, pp. 661-666, 2009.

[38] H. C. Fritts, Tree Rings and Climate, Academic Press, 1975.

[39] C. W. Stockton and H. C. Fritts, "Conditional probability of occurrence for variations in climate based on width of annual tree-rings in Arizona," Tree-Ring Bulletin, vol. 31, pp. 3-24, 1971.

[40] C. Valmore and J. R. LaMarche, "Frequency-dependent relationships between tree-ring series along an ecological gradient and some dendroclimatic implications," Tree-Ring Bulletin, vol. 34, pp. 1-20, 1974.

[41] I. Daubechies, "Recent results in wavelet applications," Journal of Electronic Imaging, vol. 7, no. 4, pp. 719-724, 1998.

[42] S. Mallat, A Wavelet Tour of Signal Processing, Academic Press, 1999.

[43] C. Torrence and G. P. Compo, "A practical guide to wavelet analysis," Bulletin of the American Meteorological Society, vol. 79, no. 1, pp. 61-78, 1998.

[44] W. J. Burroughs, Weather Cycles: Real or Imaginary? Cambridge University Press, 1994.

[45] P. S. P. Cowpertwait and A. V. Metcalfe, Introductory Time Series with $R$, Springer, 2009.

[46] M. J. Crawley, The R Book, John Wiley \& Sons, 2009.

[47] D. McCarroll, M. Tuovinen, R. Campbell et al., "A critical evaluation of multi-proxy dendroclimatology in northern Finland," Journal of Quaternary Science, vol. 26, no. 1, pp. 7-14, 2011.

[48] H. C. Fritts, "Statistical reconstruction of spatial variations in climate," in Methods of Dendrochronology: Applications in the 
Environmental Science, E. R. Cook and L. A. Kairiukstis, Eds., pp. 193-210, Kluwer Academic, Dordrecht, The Netherlands, 1990.

[49] H. C. Fritts, T. J. Blasing, B. P. Hayden, and J. E. Kutzbach, "Multivariate techniques for specifying tree-growth and climate relationships and for reconstructing anomalies in paleoclimate," Journal of Applied Meteorology, vol. 10, no. 5, pp. 845-864, 1971.

[50] G. R. Lofgren and J. H. Hunt, "Transfer functions," in Climate From Tree Rings, M. K. Hughes, P. M. Kelly, J. R. Pilcher, and V. C. LaMarche, Eds., pp. 50-56, Cambridge University Press, 1982.

[51] E. R. Cook, K. R. Briffa, and P. D. Jones, "Spatial regression methods in dendroclimatology: a review and comparison of two techniques," International Journal of Climatology, vol. 14, no. 4, pp. 379-402, 1994.

[52] J. Guiot, "Methods of calibration," in Methods of Dendrochronology: Applications in the Environmental Science, E. R. Cook and L. A. Kairiukstis, Eds., pp. 165-178, Kluwer Academic, Dordrecht, The Netherlands, 1990.

[53] H. J. Lüdecke, A. Hempelmann, and C. O. Weiss, "Multiperiodic climate dynamics: spectral analysis of long-term instrumental and proxy temperature records," Climates of the Past, vol. 9, pp. 447-453, 2013.

[54] S. Helama, M. Timonen, J. Holopainen et al., "Summer temperature variations in Lapland during the Medieval Warm Period and the Little Ice Age relative to natural instability of thermohaline circulation on multi-decadal and multi-centennial scales," Journal of Quaternary Science, vol. 24, no. 5, pp. 450-456, 2009.

[55] T. L. Delworth and M. E. Mann, "Observed and simulated multidecadal variability in the Northern Hemisphere," Climate Dynamics, vol. 16, no. 9, pp. 661-676, 2000.

[56] M. Timonen, J. Jiang, S. Helama, and K. Mielikäinen, "Significant changes of subseries-means in the Finnish tree-ring index of 7638 years, with comparisons to glaciological evidence from Greenland and Alps," Quaternary International, vol. 319, pp. 143-149, 2014.

[57] K. R. Briffa, "Annual climate variability in the Holocene: interpreting the message of ancient trees," Quaternary Science Reviews, vol. 19, no. 6, pp. 87-105, 2000.

[58] P. D. Jones, K. R. Briffa, T. P. Barnett, and S. F. B. Tett, "Highresolution palaeoclimatic records for the last millennium: interpretation, integration and comparison with General Circulation Model control-run temperatures," Holocene, vol. 8, no. 4, pp. 455-471, 1998.

[59] M. E. Mann, R. S. Bradley, and M. K. Hughes, "Northern hemisphere temperatures during the past millennium: inferences, uncertainties, and limitations," Geophysical Research Letters, vol. 26, no. 6, pp. 759-762, 1999.

[60] M. Ogurtsov, M. Lindholm, and R. Jalkanen, "Will a new little ice age begin in the next few decades?" Applied Physics Research, vol. 5, pp. 70-77, 2013.

[61] E. R. Cook, K. R. Briffa, D. M. Meko, D. A. Graybill, and G. Funkhouser, "The segment length curse' in long tree-ring chronology development for palaeoclimatic studies," Holocene, vol. 5, no. 2, pp. 229-237, 1995.

[62] R. D’Arrigo, R. Wilson, B. Liepert, and P. Cherubini, "On the "Divergence Problem" in Northern Forests: a review of the tree-ring evidence and possible causes," Global and Planetary Change, vol. 60, no. 3-4, pp. 289-305, 2008.
[63] J. Esper, D. Frank, U. Büntgen, A. Verstege, R. Hantemirov, and A. V. Kirdyanov, "Trends and uncertainties in Siberian indicators of 20th century warming," Global Change Biology, vol. 16, no. 1, pp. 386-398, 2010.

[64] S. Helama, M. Lindholm, M. Timonen, and M. Eronen, "Detection of climate signal in dendrochronological data analysis: a comparison of tree-ring standardization methods," Theoretical and Applied Climatology, vol. 79, no. 3-4, pp. 239-254, 2004.

[65] M. Lindholm and M. Eronen, "A reconstruction of midsummer temperatures from ring-widths of scots pine since $\mathrm{AD}$ 50 in northern Fennoscandia," Geografiska Annaler A: Physical Geography, vol. 82, no. 4, pp. 527-535, 2000. 

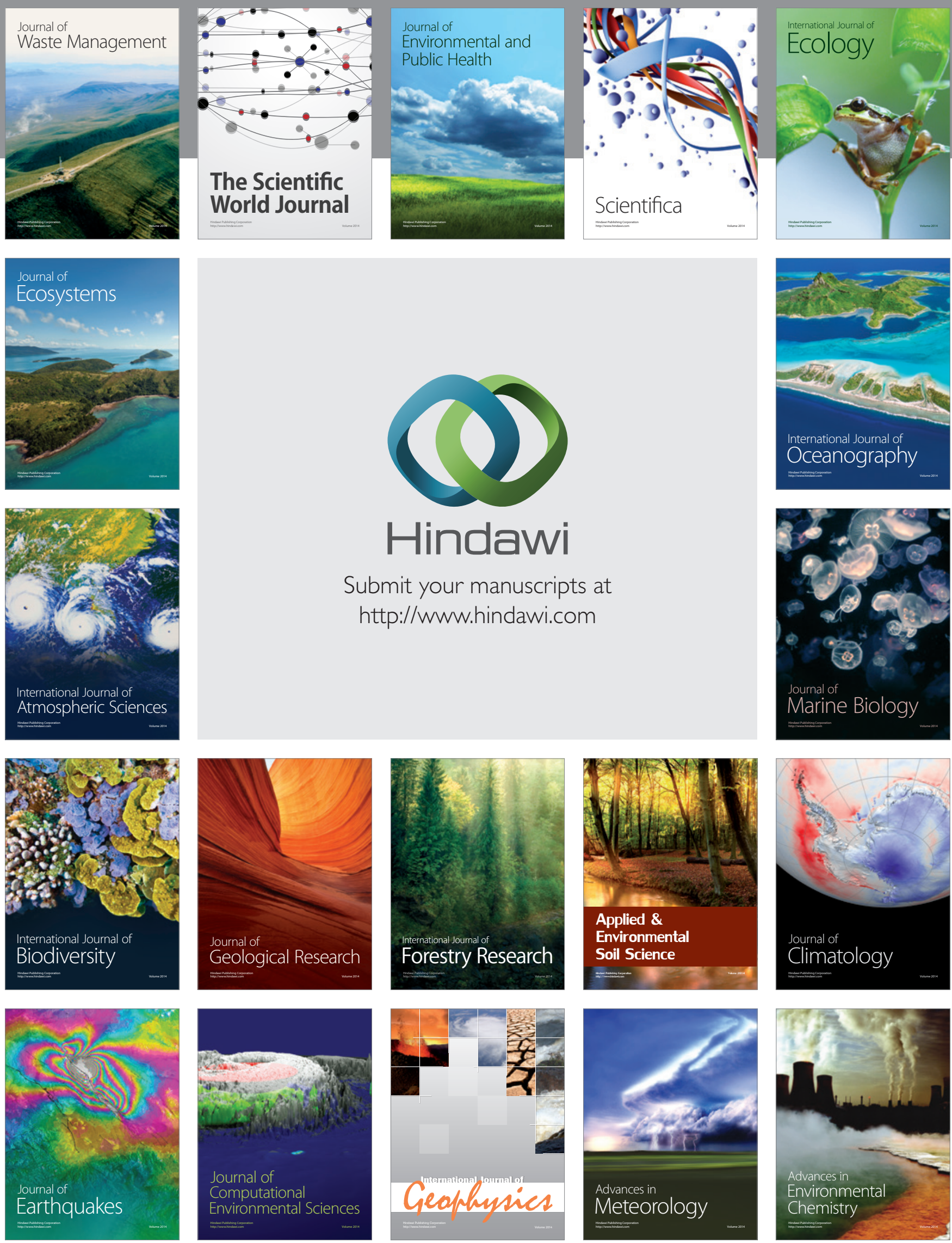